University of Nebraska - Lincoln

DigitalCommons@University of Nebraska - Lincoln

CSE Conference and Workshop Papers

Computer Science and Engineering, Department

2004

\title{
A Graph Model for DynamicWaveband Switching in WDM Mesh Networks
}

\author{
Mengke Li \\ University of Nebraska-Lincoln, mli@cse.unl.edu \\ Byrav Ramamurthy \\ University of Nebraska-Lincoln, bramamurthy2@unl.edu
}

Follow this and additional works at: https://digitalcommons.unl.edu/cseconfwork

Part of the Computer Sciences Commons

Li, Mengke and Ramamurthy, Byrav, "A Graph Model for DynamicWaveband Switching in WDM Mesh Networks" (2004). CSE Conference and Workshop Papers. 89.

https://digitalcommons.unl.edu/cseconfwork/89

This Article is brought to you for free and open access by the Computer Science and Engineering, Department of at DigitalCommons@University of Nebraska - Lincoln. It has been accepted for inclusion in CSE Conference and Workshop Papers by an authorized administrator of DigitalCommons@University of Nebraska - Lincoln. 


\title{
A Graph Model for Dynamic Waveband Switching in WDM Mesh Networks
}

\author{
Mengke Li, Student Member, IEEE, and Byrav Ramamurthy, Member, IEEE \\ Department of Computer Science and Engineering \\ University of Nebraska-Lincoln, USA \\ Email: $\{$ mli, byrav $\}$ csce.unl.edu
}

\begin{abstract}
We investigate the problem of waveband switching (WBS) in a wavelength-division multiplexing (WDM) mesh network with dynamic traffic requests. To solve the WBS problem in a homogeneous dynamic WBS network, where every node is a multi-granular optical crossconnect (MG-OXC), we construct an auxiliary graph. Based on the auxiliary graph, we develop two heuristic on-line WBS algorithms with different grouping policies, namely the wavelength-first WBS algorithm based on the auxiliary graph (WFAUG) and the waveband-first WBS algorithm based on the auxiliary graph (BFAUG). Our results show that the WFAUG algorithm outperforms the BFAUG algorithm.
\end{abstract}

\section{INTRODUCTION}

Waveband switching (WBS) refers to a technique of efficiently aggregating a set of wavelengths into a waveband at the optical crossconnects (OXCs) and transmitting them as a group. It is an important and practical technique for designing a backbone wavelength-division multiplexing (WDM) network [1] [2]. A waveband route can reduce the number of ports used compared with the corresponding wavelength routes. Thus, adopting WBS technique can generate more revenue by minimizing the switching and transmission costs in the optical domain of WDM networks, which are dominated by the number of OXC ports used. Other advantages can also be achieved, such as reducing the OXC complexity and increasing the service provisioning quality [3] [4].

A WDM network with full WBS capabilities, including waveband switching, waveband assignment, wavelength aggregation, and waveband disaggregation, is called a wavebandswitching network (WBS network) [1]. An OXC with full WBS capabilities is called a multi-granular OXC (MG-OXC). A WBS network is a homogeneous network if each node in the network is an MG-OXC. Otherwise, it is a heterogeneous network. The objective of the WBS problem is to minimize the switching and transmission costs of a WBS network given the amount of traffic. If the cost of every port in an MG-OXC is equal, the objective is to minimize the total number of used ports given the amount of traffic [4] [5].

Most previous studies on WBS have focused on networks where the traffic loads are known. The studies either analyze the performance improvement by using WBS technologies [1][4] or prove the feasibility of waveband transmission [5]. Other WBS studies have also been carried out. For example, using the GMPLS control plane to support WBS was proposed in [6], and the work in [7] studied how to design a scalable WBS network with minimum number of extra switching points.

This work was supported in part by the U. S. National Science Foundation grants (ANI-0311577 and EPS-0091900) and the UNL Program of Excellence PRISM Priority Initiative.
The previous studies on the performance analysis of WBS under static traffic requests provide evidences on the efficiency of WBS [1]- [4]. In [1], the authors propose off-line and online algorithms based on ring topology analysis. However, they relax the wavelength constraint, assuming all fibers support 160 wavelengths, and the OXC ports constraint, assuming all nodes have an infinite number of OXC ports. The relaxations are not applicable in the real WDM network design and may be crucial to the performance optimization. The static WBS problem for mesh networks has been studied in [3] with two optimal or near-optimal heuristic algorithms; an ILP formulation was proposed. Whether the heuristic algorithms are optimal in a dynamic WDM network is unknown. In [2], a classification of end-to-end WBS routing and intermediate WBS routing is proposed and the authors emphasize that their study only focuses on the end-to-end case in static WDM networks. In [4], the authors propose a non-uniform algorithm for a network carrying Zipf traffic. However, they focus on a single node case in the WBS network and do not consider the waveband routing problem. To the best of our knowledge, a detailed study of WBS with waveband routing algorithm for the dynamic WBS network has never been carried out.

In this work, we propose two on-line WBS algorithms for homogeneous dynamic WBS mesh networks based on our proposed auxiliary graphs. In an auxiliary graph, wavelength links and waveband links with various network constraints are represented by edges with property sets. The network constraints include the number of wavelengths/wavebands on each fiber-link, the number of wavelengths on each waveband route, and the wavelength conversion capabilities of the MGOXCs. The on-line WBS algorithms apply a weighted shortestpath wavelength routing algorithm or a single-hop waveband routing algorithm for each traffic demand on the auxiliary graph, and update the auxiliary graph accordingly. Different strategies for solving the dynamic WBS problem can be achieved under different WBS policies, which are the bases of the on-line WBS algorithms. We compare the performances of the two algorithms.

\section{Dynamic WBS IN WDM Mesh Networks}

\section{A. General Problem Statement}

The static WBS problem is an optimization problem. Given the network topology, traffic pattern, and traffic loads, there is an optimal scheme for grouping wavelengths into wavebands and routing them as groups with maximum cost-savings. In a real communication network, the traffic loads or patterns may change from time to time. Thus, it is challenging to find the optimal solution for the dynamic WBS problem. The inputs to the problem are as follows. 
1. $G=(V, E, W, B)$, is the physical network topology, where $V$ is a set of network nodes, $E$ is a set of fiber links connecting the nodes, $W: E \rightarrow Z^{+}$specifies the number of wavelengths on each fiber link (where $Z^{+}$denotes a set of positive integers), and $B: E \rightarrow$ $Z^{+}$specifies the number of wavebands on each fiber link. Accordingly, the capacity of a waveband $i$ is $\left\lfloor\frac{W_{i}}{B_{i}}\right\rfloor$, which means it can support $\left\lfloor\frac{W_{i}}{B_{i}}\right\rfloor$ wavelengths at most. In our study, we assume the capacities of all the wavebands are the same and each network node is an MG-OXC.

2. $\Phi=\{\varphi(s, d, t, f(t), h(t))\}$, is the set of connection requests, where $s$ is the source, $d$ is the destination, $t$ is the traffic arrival time, $f(t)$ is the interarrival time between the current traffic request and the previous one, and $h(t)$ is the request holding time. In this study, we assume each connection request $\varphi$ requires one full wavelength capacity.

3. $\Lambda=\left\{f\left(n, \lambda_{i}, \lambda_{o}\right) \mid \lambda_{i}, \lambda_{o} \in W\right\}$, is the set of wavelength conversion functions, where $n$ is the node, $\lambda_{i}$ is the input wavelength, and $\lambda_{o}$ is the output wavelength. In this study, we assume the network has no wavelength conversion ability.

4. $\Gamma=\{(n, T, R)\}$, is the set of transmitter and receiver pairs, where $n$ is the node, $T$ is the transmitter, and $R$ is the receiver. We assume each transceiver is tunable to any wavelength in the fiber.

Our goal is to determine the route for each connection request and to aggregate them into a waveband in order to minimize the number of ports occupied by the traffic, which in turn maximizes the saving ratio compared to the network without WBS capability. The online heuristic grouping policy determines when and how to aggregate the wavelengths.

\section{B. MG-OXC Architecture}

A typical MG-OXC is shown in Fig. 1 (from [1], [4]). The minor differences are reflected by the control policy. The policy control plane determines which group of wavelengths belongs to a given waveband, and which group of wavebands/wavelengths belongs to a given fiber.

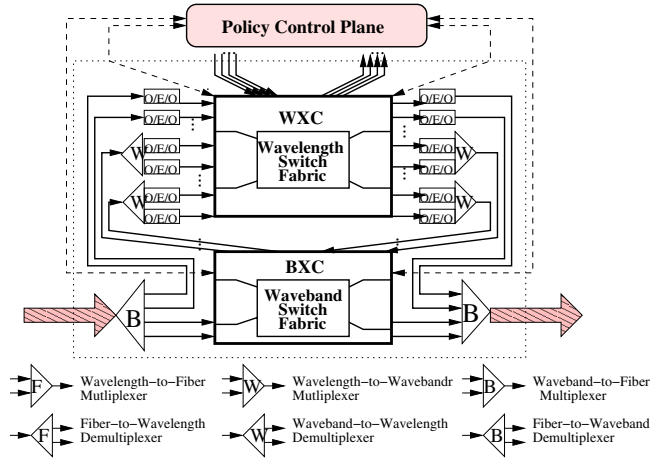

Figure 1. Multi-granular Optical Cross-Connect (MG-OXC).

In our study, the MG-OXC has no wavelength converters and arbitrary wavelengths from the same fiber can be grouped into a waveband. The MG-OXC can perform wavelengthto-waveband multiplexing, wavelength-to-fiber multiplexing, waveband-to-fiber multiplexing, waveband-to-wavelength demultiplexing, fiber-to-waveband demultiplexing, and fiber-towavelength demultiplexing. The policy control plane selects at most $m x$, possibly non-contiguous, wavelengths to be grouped into a waveband, and at most $m b$, possibly non-contiguous, wavebands to be grouped into a fiber. A fiber may contain multi-wavebands and multi-wavelengths at the same time as long as the total number of wavelengths does not exceed the fiber capacity.

\section{AuXiliary Graph CONSTRUCTION}

We construct an auxiliary graph (AUG) according to the given network configuration to assist in designing WBS algorithms. An auxiliary graph model has also been used in an earlier dynamic grooming study [8]. Our AUG is different from the one in [8] in its construction and its functionalities. Our AUG is uniquely suited for WBS networks.

Figure 2 is a simple example illustrating how to construct an AUG. Figure 2(a) is a three-node network with three unidirectional fiber links, each of which can accommodate at most 4 wavelengths and 2 wavebands. Each waveband can accommodate at most 2 wavelengths. Initially there is no connection request in the network. The AUG is constructed as in Fig. 2(b). We assume that there is no fiber switching in the network.

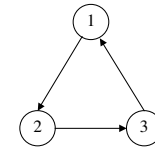

(a)

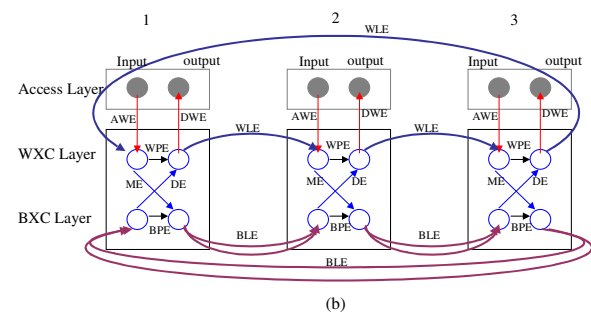

Figure 2. Auxiliary graph.
Given a network represented by a graph $G_{0}\left(V_{0}, E_{0}, W, B\right)$, we construct the corresponding auxiliary graph $A U G(V, E, \widehat{E})$ as follows, where $V$ is its vertex set, $E$ is its edge set, and $\widehat{E}$ is the set of edge properties, viz. edge identifier, edge capacity, and edge weight. The AUG is a three-layered graph, including access layer (AL), wavelength cross-connecting layer (WL), and waveband cross-connecting layer (BL) going from top to bottom. There is an input port and an output port at each layer. Traffic flow starts and terminates at the access layer at a whole wavelength capacity, and is transmitted through the other two lower layers. The edges inserted in the auxiliary graph are classified as follows.

1. Wavelength-link edges (WLE). There is an edge from the output port at the wavelength layer at node $i$ to the input port at the wavelength layer at node $j$, if there is an edge $\overrightarrow{(i, j)}$ in $G_{0}$.

2. Waveband-link edges (BLE). There is an edge from the output port at the waveband layer at node $i$ to the input port at the waveband layer at node $j$, if there is an edge $\overrightarrow{(i, j)}$ in $G_{0}$. If a BLE edge and a WLE edge 
exist from the same node $i$ to the same node $j$, they are drawn parallel to one another.

3. Multiplexing edges (ME). There is an edge from the input port at the wavelength layer to the output port at the waveband layer at node $i$, if node $i$ has wavelengthto-waveband aggregation capability.

4. Demultiplexing edges (DE). There is an edge from the input port at the waveband layer to the output port at the wavelength layer at node $i$, if node $i$ has wavebandto-wavelength disaggregation capability.

5. Add-wavelength edges (AWE). There is an edge from the transmitter to the input port at the wavelength layer at each node.

6. Drop-wavelength edges (DWE). There is an edge from the output port at the wavelength layer to the receiver at each node.

7. Wavelength-bypass edges (WPE). There is an edge from the input port to the output port at the wavelength layer at each node.

8. Waveband-bypass edges (BPE). There is an edge from the input port to the output port at the waveband layer at each node.

Each edge $e_{i}$ in the $A U G$ has an identifier $k$, a weight $w$, a capacity c, and a wavelength set $X=\{x(j) \mid j=1 . . w\}$, denoted as a property set $\widehat{e_{i}}(k, w, c)$. The identifier $k$ is used to identify the waveband route. Thus, only a BLE edge in a waveband route has a valid identifier $k$, where $k \in Z^{+}$. An invalid identifier has a value 0 . Two BLE edges contain the same identifier only if they are in the same waveband-route.

The capacity and the free wavelength set of each edge in the $A U G$ will be adjusted according to the current network state. Thus, whenever a connection is set up or torn down, the capacity and the free wavelength set of those edges along the path should be adjusted. Initially, the capacity of each WLE/WPE/AWE/DWE edge is equal to the number of wavelengths $W$ on the fiber link; the capacity of each $\mathrm{BLE} / \mathrm{BPE} / \mathrm{ME} / \mathrm{DE}$ edge is equal to the waveband capacity, which is $\left\lfloor\frac{W}{B}\right\rfloor$; every element $x(j)$ of the wavelength set for each WLE/WPE/AWE/DWE/ edge is 1, denoted as available; every element $x(j)$ of the wavelength set for each $\mathrm{DE} / \mathrm{ME} / \mathrm{BLE} / \mathrm{BPE}$ edge is 0 , denoted as not grouped. If the value of $x(j)$ is 0 on a WLE/WPE/AWE/DWE edge, it denotes that the wavelength $j$ is not available on that edge. If the value of $x(j)$ is 1 on a BLE/BPE/DW/ME edge, it denotes that the wavelength $j$ is grouped on that edge.

How the weights are assigned to each edge in the $A U G$ reflects the WBS routing policy because the routing algorithm will find a path with minimum weight for each connection among all available paths. We have two different weight assignment policies, called wavelength-first weight assignment (WFWA) and waveband-first weight assignment (BFWA). In WFWA, each ME/DE/AWE/DWE/WLE/WPE edge has a weight of 0 and each BLE/BPE edge has a weight of 1 . In BFWA, each ME/DE/AWE/DWE/BLE/BPE edge has a weight of 0 and each WLE/WPE edge has a weight of 1 . From the above, we can see that the auxiliary graph reflects the current state of the network.

\section{DyNAMIC WBS Polcies AND AlgorithmS}

\section{A. Dynamic WBS Policies}

In general, there are four possible modes to carry the traffic without altering the existing lightpaths, as shown in Fig. 3. We denote a lightpath and a waveband path with source $s$ and destination $d$ as $W(s, d)$ and $B(s, d)$ respectively. The four modes are as follows.

1. Route the traffic onto an existing waveband path $B_{i}(s, d)$, which directly connects the same source $s$ and destination $d$.

2. Set up a new lightpath $W_{N}(s, d)$ from $s$ to $d$, and route the traffic onto the lightpath.

3. Route the traffic onto two or more waveband paths $B_{1}\left(s_{1}, d_{1}\right), B_{2}\left(s_{2}, d_{2}\right), \ldots, B_{n}\left(s_{n}, d_{n}\right)$, where $s=s_{1}$, $d_{1}=s_{2}, d_{2}=s_{3}, \ldots, d_{n-1}=s_{n}$, and $d_{n}=d, n>1$.

4. Route the traffic onto one or more waveband paths $B_{1}\left(s_{1}, d_{1}\right), B_{2}\left(s_{2}, d_{2}\right), \ldots, B_{n}\left(s_{n}, d_{n}\right)$, and set up lightpaths to connect between them from $s$ to $d$.

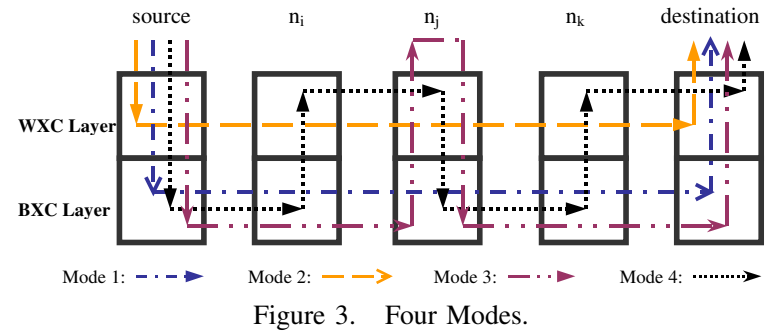

To be feasible, each mode requires some free resources. If none of the modes is feasible, the traffic request will be blocked. Different sequences of the mode adoption reflect different routing policies. In our work, we only consider the first two modes, and define two policies, called wavelengthfirst routing policy (WFRP) and waveband-first routing policy (BFRP). WFRP is based on WFWA weight assignment policy and adopts mode 1 first. BFRP is based on BFWA weight assignment policy and adopts mode 2 first.

Using the auxiliary graph, it is easy to implement these routing policies and weight assignment policies.

\section{B. Dynamic WBS Algorithms}

Based on the AUG graph and the two policies, we develop two dynamic WBS algorithms, called wavelength-first WBS algorithm based on the auxiliary graph (WFAUG) and waveband-first WBS algorithm based on the auxiliary graph (BFAUG). The algorithms take inputs and fulfill the objectives described in Section II.

Given a connection request, a dynamic WBS algorithm solves the following four subproblems: determine the wavelength/waveband route; assign wavelength; aggregate the wavelength if it can be routed in a waveband at the source node; disaggregate the wavelength if it is in a waveband at the destination node. A dynamic WBS algorithm may solve the subproblems separately or jointly. Previous studies, such as [4], only solve a few of the subproblems with a fixed route. 
In this study, we integrate all the subproblems and solve them through the algorithms.

1) Wavelength-first WBS algorithm based on the auxiliary graph (WFAUG): The WFAUG algorithm is based on WFRP policy. Before running the WFAUG algorithm, an initialization process constructs the AUG using the method discussed in Section III, assigns weight to each edge using WFWA policy, and sets $C u r_{-} B=1$, where $C u r_{-} B$ is the current free waveband identifier. The inputs are a traffic demand $\varphi(s, d, t, f(t), h(t))$ described in Section II, and the network configuration. The network configuration includes the fiber capacity $(W)$, the upper bound $(B)$ on number of wavebands in a fiber, and the upper bound $(X)$ on number of wavelengths in a waveband. The algorithm works as follows (see Fig. 4).

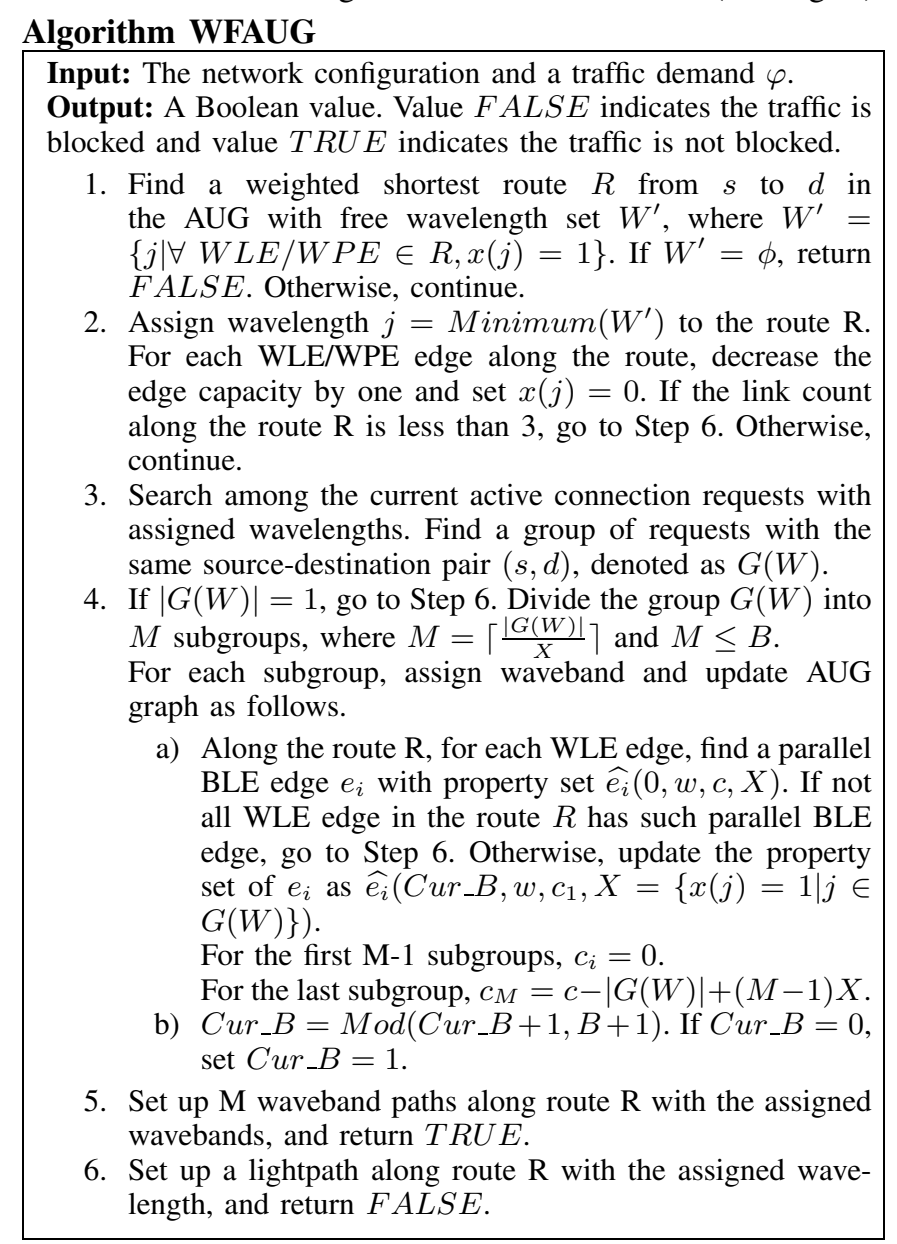

Figure 4. Description of WFAUG algorithm.

The WFAUG algorithm routes a traffic request in a wavelength path first manner. If there are multi-wavelength paths with same source and destination, it combines them into a waveband. The network state is updated according to the AUG. The WFAUG algorithm combines the weighted shortest-path algorithm, first-fit wavelength assignment algorithm, and firstfit waveband assignment algorithm, and single-hop waveband routing algorithm.

2) Waveband-first WBS algorithm based on the auxiliary graph (BFAUG): The BFAUG alogirthm is based on the BFRP policy. The initialization process constructs the AUG, assigns weight to each edge using BFWA, and sets $C u r_{-} B=1$. The inputs are a traffic demand, the network configuration, the minimum waveband utilization ratio $\eta$, and the maximum delaying time $t_{d}$. The algorithm works as follows (see Fig. 5).

\section{Algorithm BFAUG}

Input: The network configuration, a traffic demand $\varphi$, the minimum waveband utilization ratio $\eta$, and the delay $t_{d}$.

Output: A Boolean value. Value $F A L S E$ indicates the traffic is blocked and value TRUE indicates the traffic is not blocked.

1. In the AUG, find a route $R$ from $s$ to $d$ with minimum weight, where the BLE edges have the minimum freewavelength capacity greater than 0 .

2. For each BLE edges in route $R$, find the parallel WLE edge. Find a wavelength set $W^{\prime}$ such that $W^{\prime}=\{j \mid \forall W L E \in$ $R, x(j)=1\}$. If $W^{\prime}=\phi$, return $F A L S E$. Otherwise, assign wavelength $j=\operatorname{Minimum}\left(W^{\prime}\right)$ to the route R. Along the route for each WLE/WPE edge, decrease the capacity by one and set $x(j)=0$.

3. If there is a same identifier $k_{i}$ for the BLE edges along the route $\mathrm{R}$ and $k_{i} \neq 0$, continue. Otherwise, go to Step 6 .

4. Group this request into waveband $k_{i}$ and update the AUG and waveband property set as follows. Along the route R, for each BLE edge $e_{i}$ with property set $\widehat{e_{i}}\left(k_{i}, w, c_{i}, X\right)$, update $c_{i}=c_{i}-1$, set $x(j)=1$, and continue.

5. Set up a waveband path. If $C_{i} \leq(1-\eta) G$, transmit it immediately. Otherwise, after time $t_{d}$ transmit it. Return TRUE.

6. Route the connection with assigned wavelength $j$ as follows. Search the current active connection requests with assigned wavelengths. Find the first request $\varphi_{s}$ with same source $s$ and destination $d$ with assigned wavelength $i$. If there is no satisfied request, go to Step 7. Otherwise, try to group $\varphi$ and $\varphi_{s}$ into a waveband as follows.

Along the route $R$, for each WLE edge, find a parallel BLE edge $e_{i}$ with property set $\widehat{e_{i}}(0, w, c, X)$. If there is no such parallel BLE edge, go to Step 7. Otherwise, update the property set of $e_{i}$ as $\widehat{e_{i}}\left(C u r_{-} B, w, c_{i}, X\right)$, where $c_{i}=$ $c-2, C_{-} B=\operatorname{Mod}\left(C u r_{-} B+1, B+1\right), X(j)=1$, and $X(i)=1$. If $C u r_{-} B=0$, set $C u r_{-} B=1$. Return TRUE.

7. Set up a lightpath along route $R$, transmit it, and return TRUE.

Figure 5. Description of BFAUG algorithm.

The BFAUG algorithm routes a traffic request in a waveband path first manner. If no wavebands are available and there is another lightpath with the same source and destination, it combines them into a waveband. If the previous two operations fail, it routes the request in a lightpath. The network state and AUG are updated. The waveband will be routed if it reaches a given utilization level or after a given delay. The BFAUG combines the weighted shortest-path algorithm, firstfit wavelength assignment algorithm, least-capacity waveband assignment algorithm, and single-hop waveband routing algorithm.

For both the algorithms, when a connection terminates, the traffic is removed from the network. All resources are reclaimed and the AUG is updated.

\section{Results And Discussion}

In this section, we evaluate the two proposed dynamic WBS algorithms, WFAUG and BFAUG, on the NSF backbone 
network [3], which consists of 14 MG-OXC nodes and 21 bidirectional links. The wavelengths are routed in full capacities, and the capacities of all wavebands/fibers are the same. In our dynamic network simulation, the traffic arrival process is Poisson and the traffic holding time follows a negative exponential distribution. The traffic is uniformly distributed among all node pairs.

We compare the performances of the WFAUG and BFAUG algorithms under different network loads in terms of Erlang: $L \in\{70,80,90,100\}$, with different wavelength capacities per fiber link: $W \in\{8,16,32,64,160\}$, and with different wavelength capacities per waveband: $G \in\{2,3,4,6,8\}$. Under each scenario and for each WBS algorithm, we simulate 100,000 connection requests to obtain the performance. The waveband utilization ratio $\eta$ in the BFAUG algorithm ranges from $50 \%$ to $100 \%$. The metric used in the simulation is the number of ports saved under dynamic WBS operation. We also calculate the ratio of wavelength ports to waveband ports and show the real cost saving ratio for both algorithms. Under all scenarios, the WFAUG algorithm outperforms the BFAUG algorithm. This is reasonable because BFAUG will not transmit the connection in a waveband if the waveband utilization is low.

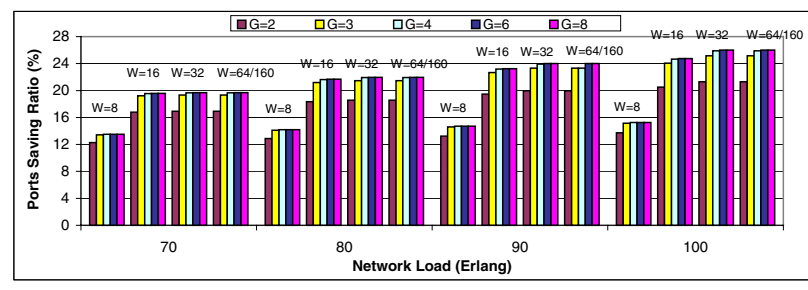

Figure 6. Port saving ratio of WFAUG.

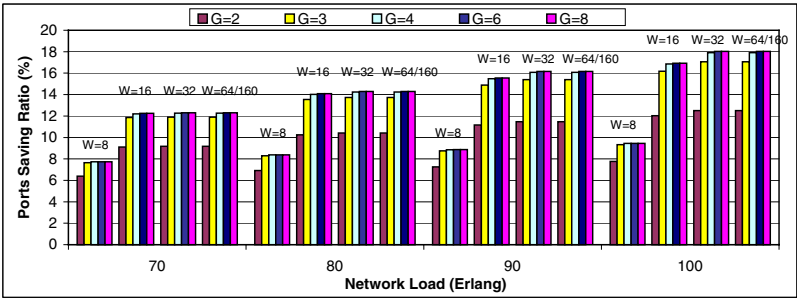

Figure 7. Average port saving ratio of BFAUG for $\eta=60 \%, \eta=70 \%$, and $\eta=80 \%$.

The port saving ratio obtained from $W F A U G$ is shown in Fig.6 and the port saving ratio obtained from $B F A U G$ is shown in Fig.7. In both algorithms, as the waveband capacity increases the port saving ratio increases; as the number of wavelengths in a fiber increases the port saving ratio increases. The reason for the increase in the port saving ratio is the increase in the number of connections over waveband routes. The increasing rate slows down as the waveband capacity reaches 6 and the number of wavelengths in a fiber reaches 32 . For the case when waveband capacity is greater than 6 and fiber capacity greater than 32, the port saving ratio remains approximately at $26 \%$ for the WFAUG algorithm and at $18 \%$ for the BFAUG algorithm. By increasing the number of wavelengths in a fiber link to 32 , both algorithms show that all connection requests can be provisioned.
To illustrate that the real cost saving might be different from the port saving ratio, we provide the results for the following case. The network load ranges from 70 Erlangs to 100 Erlangs. The fiber capacity is 64 and the waveband capacity ranges from 2 to 8 . Assume only waveband switching ports are alloptical OXC ports and the cost of an $\mathrm{O} / \mathrm{O} / \mathrm{O}$ port is only $\frac{1}{5}$ of an $\mathrm{O} / \mathrm{E} / \mathrm{O}$ port. The results on the ratio of wavelength ports to waveband ports is shown in Fig. 8 for both the WFAUG algorithm and the BFAUG algorithm. The WFAUG algorithm still outperforms the BFAUG algorithm. However, the difference in the performance of the WFAUG and BFAUG algorithms for the real cost saving is less than the one for the port saving ratio.

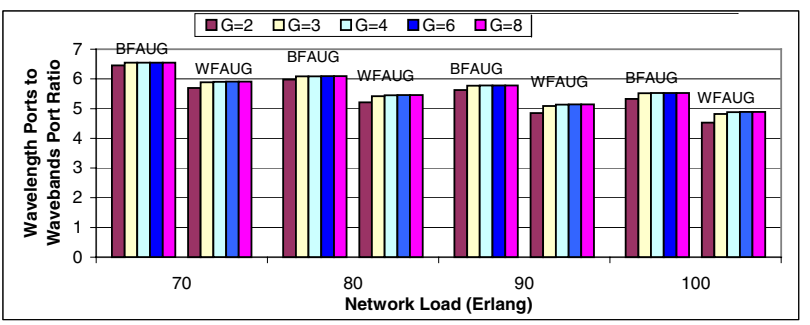

Figure 8. Ratio of wavelength ports to waveband ports for the NSF network $(\mathrm{W}=64)$.

\section{CONCLUSION}

We formulated the dynamic WBS problem in a WDM mesh network and proposed to use the auxiliary graph model to assist dynamic WBS algorithm design. We constructed the auxiliary graph given the network configuration. Based on the auxiliary graph, we proposed two dynamic WBS algorithms. Simulation results showed that the WFAUG algorithm outperforms the BFAUG algorithm. In future, we plan to utilize the auxiliary graph to assist in the designing of a dynamic WBS algorithm using multi-hop waveband routing for a heterogeneous network and test the dynamic WBS algorithms for other scenarios.

\section{REFERENCES}

[1] Y. Suemura, I. Nishioka, Y. Maeno, S. Araki, R. Izmailov, and S. Ganguly, "Hierarchical routing in layered ring and mesh optical networks," Proc. IEEE ICC'02, vol. 5, pp. 2727-2733, Apr. 2002.

[2] L. Noirie, M. Vigoureux, and E. Dotaro, "Impact of intermediate grouping on the dimensioning of multi-granularity optical networks," Proc. of Optical Fiber Communication (OFC'01), pp. TuG3/1-3, Mar. 2001.

[3] X. Cao, V.Anand, Y. Xiong, and C. Qiao, "Performance evaluation of waveblength band switching in multi-fiber all-optical networks," Proc. IEEE INFOCOM '03, vol. 3, pp. 2251-2261, Apr. 2003.

[4] I. Rauf, G. Samrar, K. Viktor, and C. Aikaterini, "Non-uniform waveband hierarchy in hybrid optical networks," Proc. IEEE INFOCOM'2003, vol. 2, pp. 1344-1354, Apr. 2003.

[5] P. Toliver, R. Runser, J. Young, and J. Jackel, "Experimental field trial of waveband switching and transmission in a transparent reconfigurable optical network," Proc. of Optical Fiber Communication (OFC'03), vol. 2, pp. 783-784, Apr. 2003.

[6] R. Douville, D. Papadimitriou, E. Dotaro, R. Izmailov, A. Kolarov, and J. Drake, "Extensions to generalized MPLS in support of waveband switching," Internet draft, draft-douville-ccamp-gmpls-wavebandextensions-04.txt, June 2003.

[7] P. Ho, H. Mouftah, and J. Wu, "A novel designing of optical crossconnects with multi-granularity provisioning support for the nextgeneration internet," Proc. IEEE ICC'03, vol. 1, pp. 582-587, May 2003.

[8] H.Zhu, H. Zang, K. Zhu, and B. Mukherjee, "A novel generic graph model for traffic grooming in heterogeneous WDM mesh networks," IEEE/ACM Transactions on Networking, vol. 11, no. 2, pp. 285-299, Apr. 2003. 